\title{
Antifungal Activity of Lactobacillus pentosus LOCK 0979 in the Presence of Polyols and Galactosyl-Polyols
}

\author{
Lidia Lipińska $^{1} \cdot$ Robert Klewicki $^{2} \cdot$ Michal Sójka $^{2} \cdot$ Radosław Bonikowski $^{3}$. \\ Dorota Żyżelewicz $^{2}$ - Krzysztof Kolodziejczyk ${ }^{2} \cdot$ Elżbieta Klewicka $^{1}$
}

Published online: 6 November 2017

(C) The Author(s) 2017. This article is an open access publication

\begin{abstract}
The antifungal activity of Lactobacillus pentosus ŁOCK 0979 depends both on the culture medium and on the fungal species. In the control medium, the strain exhibited limited antagonistic activity against indicator food-borne molds and yeasts. However, the supplementation of the bacterial culture medium with polyols (erythritol, lactitol, maltitol, mannitol, sorbitol, xylitol) or their galactosyl derivatives (gal-erythritol, gal-sorbitol, gal-xylitol) enhanced the antifungal properties of Lactobacillus pentosus ŁOCK 0979. Its metabolites were identified and quantified by enzymatic methods, HPLC, UHPLC-MS coupled with QuEChERS, and GC-MS. The presence of polyols and gal-polyols significantly affected the acid metabolite profile of the bacterial culture supernatant. In addition, lactitol and mannitol were used by bacteria as alternative carbon sources. A number of compounds with potential antifungal properties were identified, such as phenyllactic acid, hydroxyphenyllactic acid, and benzoic acid. Lactobacillus bacteria cultivated with mannitol synthesized hydroxy-fatty acids, including 2-hydroxy-4methylpentanoic acid, a well-described antifungal agent. Scanning electron microscopy (SEM) and light microscopy confirmed a strong antifungal effect of $L$. pentosus $Ł O C K$ 0979.
\end{abstract}

Lidia Lipińska

lidialipinska8@gmail.com

1 Lodz University of Technology, Institute of Fermentation Technology and Microbiology, Wolczanska 171/173, 90-024 Lodz, Poland

2 Lodz University of Technology, Institute of Food Technology and Analysis, Stefanowskiego 4/10, 90-024 Lodz, Poland

3 Lodz University of Technology, Institute of General Food Chemistry, Stefanowskiego 4/10, 90-024 Lodz, Poland
Keywords Antifungal activity · Galactosyl-polyols · Lactobacillus $\cdot$ Metabolites $\cdot$ Polyols $\cdot$ SEM

\section{Introduction}

Filamentous fungi and yeasts are present in almost all types of ecosystems due to their high adaptation ability and low nutritional requirements. Filamentous fungi are widespread food spoilage microorganisms responsible for significant economic losses in the agri-food industry [6]; they are also a major health concern due to mycotoxin production. The most common genera of spoilage fungi include Penicillium, Fusarium, Aspergillus, Cladosporium, and Rhizopus [21]. Commercial foodstuffs are usually protected from such microorganisms by physical and chemical techniques. However, as chemical preservatives have become less socially acceptable, natural preservation methods are being sought. Lactic acid fermentation has been known and used these purposes since antiquity. In recent years, lactic acid bacteria (LAB) have been extensively investigated for their antifungal properties and bioprotective cultures have been proposed as a promising biotechnological approach [22, 24, 25]. Of particular application interest are lactobacilli, which convert carbohydrates into lactic and acetic acids (primary metabolites), as well as a range of secondary metabolites, such as carbon dioxide, ethanol, hydrogen peroxide, fatty acids, acetoin, diacetyl, cyclic dipeptides, bacteriocins, and bacteriocin-like inhibitory substances [3]. Since these metabolites exhibit only weak antifungal properties, many research teams are seeking Lactobacillus strains with a higher natural ability to inhibit fungal and yeast growth $[4,9$, 14, 15, 26]. Ryu et al. [26] reported that Lactobacillus plantarum HD1 synthesizes 5-oxododecanoic acid (MW 214), 3-hydroxydecanoic acid (MW 188), and 3-hydroxy-5dodecenoic acid (MW 214), which are considered antifungal. 
In turn, Magnusson $[14,16]$ showed that some LAB can convert glycerol to 1,3-propanediol, which inhibits fungal growth. While the qualitative and quantitative composition of antifungal compounds generated by LAB is species- or even strain-specific, it can be modulated by culture medium modification. For instance, Lipińska et al. [13] adjusted the antifungal spectrum of lactobacilli by adding polyols and their galactosyl derivatives, proving that the antagonistic activity of $\mathrm{LAB}$ depends on culture medium composition, the LAB species, and the sensitivity of the fungal species. It was found that in the presence of xylitol and gal-xylitol in the bacterial culture medium Lactobacillus pentosus ŁOCK 0979 effectively inhibited the growth of A. niger, A. alternata, A. brassicicola, F. lateritium, and M. hiemalis [13]. The modulation of LAB metabolism by supplementing the culture medium with various, often atypical, compounds may give rise to new systems inhibiting the growth of spoilage microorganisms.

The objective of the study was to determine the antifungal properties, metabolite profile, and enzymatic activity of the strain L. pentosus ŁOCK 0979 cultured in the presence of polyols, namely, erythritol, xylitol, maltitol, mannitol, sorbitol, and lactitol, and their transglycosylation derivatives (galerythritol, gal-xylitol, and gal-sorbitol).

\section{Materials and Methods}

\section{Microbiological Strains and Polyols}

The study material consisted of the bacterial strain $L$. pentosus ŁOCK 0979 and 10 fungal strains deposited with the Pure Cultures Collection of Industrial Microorganisms of the Institute of Fermentation Technology and Microbiology, Lodz University of Technology (LOCK 105). The indicator fungi included the yeasts Candida vini 0008 and 0009 and the molds Mucor hiemalis 0519, Geotrichum candidum 0511, Alternaria alternata 0409, Alternaria brassicicola 0412, Aspergillus niger 0433, Fusarium lateritium 0508, Aspergillus ochraceus, and Penicillium sp. Two of the tested fungi, A. ochraceus and Penicillium sp., were newly isolated from spoiled food.

Fungi were grown in Sabouraud 4\% dextrose agar (Merck) and bacteria in MRS medium (Merck) supplemented with $1 \%$ $(\mathrm{m} / \mathrm{v})$ polyols (erythritol, lactitol, maltitol, mannitol, sorbitol, xylitol) or galactosyl polyols (gal-erythritol, gal-sorbitol, galxylitol). The microorganisms were cultured at $30{ }^{\circ} \mathrm{C}$ under aerobic conditions. Fungi were stored at $4{ }^{\circ} \mathrm{C}$ on Sabouraud dextrose agar slants (Merck), and bacteria were kept at $-20{ }^{\circ} \mathrm{C}$ in $20 \%(v / v)$ glycerol.

The polyols used in the study (erythritol, lactitol, maltitol, mannitol, sorbitol, xylitol) occur naturally in some foodstuffs and may be added to others, e.g., as sweeteners. In turn, gal- erythritol, gal-xylitol, and gal-sorbitol are modern prebiotics which confer beneficial effects [8], as related in the blood and digesta of laboratory rats (Klewicki 2007).

\section{Synthesis of Galactosyl Derivatives of Erythritol, Sorbitol, and Xylitol}

Galactosyl derivatives of erythritol, sorbitol, and xylitol were obtained by enzymatic transglycosylation using $\beta$ galactosidase EC 3.2.1.23 from Kluyveromyces lactis (Novozymes A/S, Bagsvaerd, Denmark). The procedure for galactosyl-xylitol synthesis was described by Klewicki [11].

\section{Determination of Antifungal Activity of Lactobacillus pentosus LOCK 0979 in the Presence of Polyols and Galactosyl-Polyols}

The antagonistic activity of $L$. pentosus ŁOCK 0979 against the indicator fungi was tested using the double-layer method described by Lipińska et al. [13]. First, $10 \mu \mathrm{L}$ of overnight bacterial culture was dropped on MRS agar plates (Merck or BTL) supplemented with $1 \%(\mathrm{~m} / \mathrm{v})$ polyols, galactosylpolyols, or galactose, separately. The control group consisted of MRS agar plates (Merck) with lactobacilli colonies cultured with neither polyols nor gal-polyols. After 18-24 h, the plates were overlaid with Sabouraud 4\% dextrose agar (Merck) inoculated with an indicator fungal strain $\left(10^{5}-10^{6}\right.$ spores $\left.\times \mathrm{mL}^{-1}\right)$. Indicator strain inhibition zones around Lactobacillus sp. colonies were measured after $24-72 \mathrm{~h}$ of cultivation at $30^{\circ} \mathrm{C}$. The results were given as fungal inhibition diameters minus the diameter of Lactobacillus sp. colonies.

\section{Preparation of Cell-Free Supernatant After Lactic Acid Fermentation}

Following lactic acid fermentation by L. pentosus LOCK 0979 in media with one of polyols or galactosyl-polyols, samples of cell-free supernatant (CFS) were prepared in order to identify and quantify the antifungal agents produced by the bacteria in the modified MRS media.

The media consisted of MRS broth (Merck) containing 1\% $(\mathrm{m} / \mathrm{v})$ glucose supplemented with $1 \%(\mathrm{~m} / \mathrm{v})$ of one of the polyols (erythritol, lactitol, maltitol, mannitol, sorbitol, or xylitol) or one of the gal-polyols (gal-erythritol, gal-sorbitol, or gal-xylitol). The final $\mathrm{pH}$ was $5.7 \pm 0.2$. In the first step of the experiment, $200 \mathrm{~mL}$ of a medium was inoculated with $3 \%(\mathrm{v} /$ $v)$ of overnight $L$. pentosus ŁOCK 0979 culture $\left(10^{5}\right.$ $\left.10^{6} \mathrm{cfu} \times \mathrm{mL}^{-1}\right)$, and incubated for $48 \mathrm{~h}$ at $30{ }^{\circ} \mathrm{C}$. Subsequently, the samples were centrifuged $(10 \mathrm{~min}$, $12,000 \times g, 20^{\circ} \mathrm{C}$ ), and the supernatants were filtered using $0.22-\mu \mathrm{m}$ syringe filters. $\mathrm{CFS}$ were stored at $-20^{\circ} \mathrm{C}$ for further study. 


\section{Determination of the Content of Polyols and Saccharides Using HPLC}

Each CFS sample was diluted 10-fold. The obtained solution was passed through a $5-\mathrm{mL}$ column BAKERBOND® spe Octadecyl (18) (J.T. Baker, USA) with cation and anion exchange resins $(1: 2 v / v)$. The first fraction $(3 \mathrm{~mL})$ was discarded, and the second one $(3 \mathrm{~mL})$ was collected for HPLC analysis. The content of saccharides and polyols was determined using an Aminex HPX-87C column from Bio-Rad $(0.78 \times 30 \mathrm{~cm}$, mobile phase: water, flow rate: $0.5 \mathrm{~mL} \times \min ^{-1}, 85^{\circ} \mathrm{C}$ ). An RI detector and an integrating system from Knauer were used. The tests were done in triplicate and prepared in three parallel columns. Statistical analysis consisted of the Duncan test $(p \leq 0.05)$.

\section{Spectrophotometric Determination of Glucose}

Glucose concentration in the pure culture medium and in postfermentation CFS was determined spectrophotometrically according to the instructions supplied with the enzymatic kit (BioMaxima) and a calibration curve. CFS and pure culture medium samples were diluted 50- and 100-fold, respectively, relative to the initial glucose content of the medium $\left(10 \mathrm{~g} \times \mathrm{L}^{-1}\right)$. Subsequently, $1 \mathrm{~mL}$ of the reagent (glucose oxidase and glucose peroxidase) and $0.01 \mathrm{~mL}$ of a tested sample (diluted culture or pure medium) were placed in a cuvette and mixed. After $5 \min \left(37^{\circ} \mathrm{C}\right)$, the absorbance of the tested sample was measured relative to the reagent blank $(\lambda=540 \mathrm{~nm}$ ), with the results being proportionate to glucose content in the sample. Based on the prepared calibration curve (in the range of $0.01-4 \mathrm{~g}$ glucose $\times \mathrm{L}^{-1}$ ) glucose concentration was determined both in medium and CFS samples, accounting for their dilution. The tests for each sample were done in triplicate, and statistical analysis involved one-way ANOVA $(p \leq 0.05)$.

\section{Concentration of D-Lactic Acid, L-Lactic Acid, and Acetic Acid}

The quantification of D-lactic, L-lactic, and acetic acid requires enzymatic reactions described in the assay procedures: $\mathrm{K}$ DLLATE $07 / 14$ and K-ACET 11/05 (Megazyme International Ireland). In the case of D- and L-lactic acids, the manufacturer's procedure for the sequential assay of both optical isomers was applied. The concentration of all tested acids was estimated using colorimetric tests with the absorbance measured $(\lambda=340 \mathrm{~nm})$ in a control sample (non-inoculated medium) and diluted CFS. The calculations were made according to the manufacturer's recommendations, taking into consideration the dilution factor $(\mathrm{F}=50)$. The tests were done in triplicate, and statistical analysis involved one-way ANOVA $(p \leq 0.05)$.

\section{Quantification of Antifungal Acids Using UHPLC-MS in Conjunction with QuEChERS}

Antifungal metabolites produced by L. pentosus ŁOCK 0979 in the presence of polyols and their galactosyl derivatives were quantified using the QuEChERS method and an ultra-highperformance liquid chromatography-mass spectrometry (UHPLC-MS) system according to a protocol modified from Oliveira et al. [20]. In the sample preparation step, $1 \mathrm{~mL}$ of formic acid, $10 \mathrm{~mL}$ of ethyl acetate, and $10 \mathrm{~mL}$ of a CFS sample were added to a Falcon test tube containing $4 \mathrm{mg}$ of magnesium sulfate and $1 \mathrm{~g}$ of sodium chloride. The mixture was shaken for $1 \mathrm{~min}$ and centrifuged $(10 \mathrm{~min}, 1077 \times \mathrm{g})$. Then $5 \mathrm{~mL}$ of the organic solvent was removed and added to an Agilent dSPE kit (150 mg of C18, $900 \mathrm{mg}$ of magnesium sulfate). The mixture was shaken for $1 \mathrm{~min}$, centrifuged $(10 \mathrm{~min}, 1077 \times \mathrm{g})$, and decanted into a test tube with $100 \mathrm{~mL}$ of dimethyl sulfoxide (DMSO). The solutions were concentrated for $3.5 \mathrm{~h}$ in a ScanVac ScanSpeed 40 centrifuge evaporator $\left(2000 \mathrm{rpm}, 45^{\circ} \mathrm{C}\right)$ equipped with a CoolSafe 110-4 Pro cold trap (Labogene, Lynge, Denmark) until only $100 \mu \mathrm{L}$ DMSO remained. The concentrated solution was mixed with $400 \mu \mathrm{L}$ of $10 \%(v / v)$ acetonitrile, centrifuged $(10 \mathrm{~min}$, $10,000 \times g$ ), and transferred into a $1.5-\mathrm{mL}$ amber vial.

The following 13 antifungal compounds were quantified: DL-3-phenyllactic acid, DL-p-hydroxyphenyllactic acid, benzoic acid, hydrocaffeic acid, hydrocinnamic acid, vanillic acid, 4-hydroxybenzoic acid, catechol, caffeic acid, ferulic acid, 3-hydroxybenzoic acid, 2,4-dihydroxybenzoic acid, and p-coumaric acid.

A Dionex UltiMate 3000 ultra-high-performance liquid chromatograph from Thermo Fisher Scientific (Germering, Germany) coupled with a diode array detector (DAD) and a Q Exactive Orbitrap mass spectrometer (MS, Thermo Fisher Scientific, Bremen, Germany) was used for LC-MS analysis. Chromatographic separation was performed using a $150-\mathrm{mm}$ C18 column with a $2.1-\mathrm{mm}$ internal diameter and $2.6-\mu \mathrm{m}$ particle size (Kinetex 2.6u, Torrance, CA, USA). The column temperature was maintained at $30^{\circ} \mathrm{C}$, and the injection volume was $2.5 \mu \mathrm{L}$. The mobile phase consisted of the following: A was water containing $0.1 \%$ formic acid and B was a mixture of acetonitrile and water $(90: 10, v / v)$ containing $0.1 \%$ formic acid. The flow rate was $0.5 \mathrm{~mL} / \mathrm{min}$. The following gradient was used: 0-16.5 min, 5-40\% B; 16.5-17.5 min, 40-95\% B; 17.5-20 min, 95\% B; 20-22 min, 95-5\% B; 22-27 min, 5\% B. After DAD detection, the separated compounds entered into the MS system via a heated electrospray ionization $(\mathrm{H}-$ ESI) source with a flow rate of $0.5 \mathrm{~mL} / \mathrm{min}$. Analyses were carried out in the negative ion mode. Chromatographic data were collected using Xcalibur software (Thermo). The source parameters were as follows: a vaporizer temperature of $400{ }^{\circ} \mathrm{C}$, an ion spray voltage of $4 \mathrm{kV}$, a capillary temperature of $380^{\circ} \mathrm{C}$, and sheath and auxiliary gas flow rates of 60 and 
15 units, respectively. The detector was operated in either full MS or full MS/dd-MS2 scan modes. In the full MS mode, the scan rage of $m / z 50-400$ was used. The full MS/dd-MS ${ }^{2}$ scan mode was used to generate MS2 data. In this mode, the selected precursor ions entered into a high-energy collision-induced dissociation (CID) cell, where they were fragmented with normalized collision energy (NCE) to obtain product ion spectra $\left(\mathrm{MS}^{2}\right)$. In our experiments, the NCE used to generate $\mathrm{MS}^{2}$ spectra was set to 30 . Tuning and optimization were performed using direct injection of the standard solution diluted in an 80:20 $(v / v)$ mixture of mobile phases A and B at a flow rate of $0.25 \mathrm{~mL} / \mathrm{min}$. Acids were quantified using the selected ion monitoring (SIM) mode. The standard curves of these compounds were used for quantification. Table 1 gives acquisition parameters for 13 acids in the tested solution.

Acid quantification was performed in triplicate, and statistical analysis was conducted using the Duncan test $(p \leq 0.05)$.

\section{Identification of Fatty Acids and Hydroxylated Fatty Acids by Gas Chromatography Coupled with Mass Spectrometry}

Lactobacillus pentosus ŁOCK 0979 was grown at $30{ }^{\circ} \mathrm{C}$ in $150 \mathrm{~mL}$ of MRS broth (Merck) with $1 \%(\mathrm{~m} / \mathrm{v})$ mannitol. The 48 -h culture was centrifuged $\left(10 \mathrm{~min}, 12,000 \times \mathrm{g}, 20^{\circ} \mathrm{C}\right.$ ), and supernatant $\mathrm{pH}$ was adjusted to 4.0 with hydrochloric acid. Then, $100 \mathrm{~mL}$ of the sample was extracted with $30 \mathrm{~mL}$ of dichloromethane, mixed for $3 \mathrm{~min}$, and settled for $10 \mathrm{~min}$. Extraction of the aqueous phase was repeated twice using further portions of dichloromethane. The organic phases were combined, dried over anhydrous sodium sulfate, and, following filtration, concentrated to approx. $0.5 \mathrm{~mL}$ in a rotatory evaporator. The residue was derivatized with $200 \mu \mathrm{L}$ of $0.25 \mathrm{M}$ trimethylsulfonium hydroxide solution (TMSH, Sigma Aldrich) in methanol.
The samples were analyzed by gas chromatography coupled with mass spectrometry (TRACE GC Ultra-ISQ) using a Stabilwax-DA capillary column $(30 \mathrm{~m} \times 0.25 \mathrm{~mm}$ i.d., film thickness $0.25 \mu \mathrm{m})$. The operating conditions were as follows: temperature program $-50{ }^{\circ} \mathrm{C}(3 \mathrm{~min})-240{ }^{\circ} \mathrm{C}$ (30 min) at $4{ }^{\circ} \mathrm{C} / \mathrm{min}$, injection temperature $-240{ }^{\circ} \mathrm{C}$, carrier gas-helium (constant flow $1 \mathrm{~mL} / \mathrm{min}$ ). Mass spectrometer parameters were as follows: $33-550 \mathrm{amu}$, ionization energy $70 \mathrm{eV}$, ion source temperature $200{ }^{\circ} \mathrm{C}$. Identification of compounds was based on a comparison of their mass spectra with computerized libraries (Wiley Registry 10th Edition/NIST Mass Spectral Library 2012).

Mannitol was chosen as one of the best agents enhancing the antifungal effect of L. pentosus ŁOCK 0979. Moreover, in the presence of mannitol, many signals from acidic compounds were obtained using UHPLC-MS analysis, but they could not be identified by the UHPLC-MS method due to their molecular structure (data not presented).

\section{API®ZYM 25200 Test of Bacterial Enzymatic Activity}

Bacteria were grown for $24 \mathrm{~h}$ in $9 \mathrm{~mL}$ of MRS broth (Merck) with $1 \%(\mathrm{~m} / \mathrm{v})$ polyols or galactosyl-polyols, added one by one. The cultures were centrifuged (10 min, $12,000 \times \mathrm{g}, 20^{\circ} \mathrm{C}$ ), and the biomass was suspended in saline to obtain a cell concentration corresponding to 5-6 on the McFarland scale (approx. $1.5 \times 10^{9}$ cells $\times \mathrm{mL}^{-1}$ ). API ZYM strips were placed in API ZYM boxes humidified by distilled water. The strips were inoculated with $65 \mu \mathrm{L}$ of the sample and incubated for $4 \mathrm{~h}$ at $37{ }^{\circ} \mathrm{C}$. Then, the reagents ZYM A and ZYM B (bioMerieux) were added dropwise. The strips were placed under a powerful light source for $10 \mathrm{~s}$ and then exposed to daylight for 5 $10 \mathrm{~min}$. Results were read according to the manufacturer's recommendations.
Table 1 LC-MS acquisition parameters for acids detected in post-fermentation cell-free supernatant

\begin{tabular}{llll}
\hline Compound & Retention time $(\mathrm{min})$ & Confirmation ion $(\mathrm{m} / \mathrm{z})$ & Quantitation ion $(\mathrm{m} / \mathrm{z})$ \\
\hline Catechol & 3.27 & - & 109 \\
DL- -Hydroxyphenyllactic acid & 3.44 & $163,135,119$ & 181 \\
3-Hydroxybenzoic acid & 4.38 & 93 & 137 \\
Hydrocaffeic acid & 4.88 & 137,109 & 181 \\
2,4-Dihydroxybenzoic acid & 5.47 & 109 & 153 \\
4-Hydroxybenzoic acid & 5.47 & 93 & 137 \\
Vanillic acid & 5.52 & $152,123,108$ & 167 \\
Caffeic acid & 5.88 & 135 & 179 \\
DL-3-Phenyllactic acid & 6.80 & 147,119 & 165 \\
$p$-Coumaric acid & 7.88 & 119 & 163 \\
Ferulic acid & 8.74 & 178,134 & 193 \\
Hydrocinnamic acid & 12.17 & 121 & 149 \\
\hline
\end{tabular}


The Effects of Cell-Free Supernatants on Fungal Growth and Morphology Evaluated with Scanning Electron Microscopy and Light Microscopy

Fungal strains sensitive to the metabolites of polyols or galpolyols were selected based on the antagonistic activity of L. pentosus ŁOCK 0979 cultured in different culture media. CFS samples were added to Sabouraud 4\% dextrose agar (Merck) in the amount of $10 \%(v / v)$. The medium was subsequently placed in 6-well plates and inoculated with selected fungal strains using an inoculation loop. Microscopic examination was carried out after 2 days (yeasts) and 7 days (molds) using a light microscope. Additionally, yeast morphology was examined using a scanning electron microscope (JEOL JCM6000, Tokyo, Japan) after coating with gold particles for $45 \mathrm{~s}$ (JEOL JFC-1200 Fine Coater, Tokyo, Japan). The experiments were conducted in duplicate. The control samples consisted of fungi cultured on Sabouraud agar without bacterial CFS.

\section{Results}

\section{Antifungal Activity of Lactobacillus pentosus LOCK 0979 in the Presence of Polyols and Galactosyl-Polyols}

The antagonistic activity of L. pentosus ŁOCK 0979 against the tested yeasts was weak, but its anticandidal properties were enhanced in the presence of galactosyl-polyols, and especially gal-erythritol. The addition of gal-sorbitol and gal-xylitol to the bacterial culture medium led to inhibition of only one of the two strains of yeast, that is, C. vini ŁOCK 0009 (Table 2).
Mold inhibition by L. pentosus ŁOCK 0979 depended both on the culture medium composition and on mold species. The A. alternata test strain and the A. ochraceus and Penicillium sp. strains isolated from the environment exhibited the greatest sensitivity to lactic acid fermentation products both in the controls and in samples with polyols and gal-polyols (Table 2). In contrast, the growth of A. brassicicola and A. niger was inhibited by L. pentosus LOCK 0979 only if the bacterial culture medium was supplemented with polyols (both mold strains) or gal-polyols (only A. brassicicola). The antifungal activity of $L$. pentosus ŁOCK 0979 was also improved by polyols and their galactosyl derivatives with respect to F. lateritium. Its growth was inhibited by the bacteria cultured in the presence of maltitol and sorbitol, as well as all tested galactosyl-polyols. Antagonistic activity against G. candidum and M. hiemalis was weak (Table 2).

In a similar way, additional control trials were conducted using MRS medium with glucose (Merck) and $1 \%(\mathrm{~m} / \mathrm{v})$ galactose, as well as a glucose-free MRS medium (BTL) with $1 \%(\mathrm{~m} /$ $v)$ galactose. These media enhanced the antagonistic activity of L. pentosus ŁOCK 0979 only against one indicator fungal strain (F. lateritium) as compared to bacteria cultivated on MRS agar (Merck). Therefore, it can be assumed that the small amounts of galactose released as a result of galactosyl-polyol hydrolysis (Table 3) are not a critical determinant of antifungal properties.

\section{Content of Polyols and Saccharides in Cell-Free Supernatant}

The content of polyols and saccharides before and after lactic acid fermentation by L. pentosus ŁOCK 0979 in the presence of
Table 2 Antifungal activity of Lactobacillus pentosus ŁOCK 0979

\begin{tabular}{|c|c|c|c|c|c|c|c|c|c|c|}
\hline \multirow[t]{2}{*}{ Growth media } & \multicolumn{2}{|c|}{ Yeasts } & \multicolumn{8}{|c|}{ Molds } \\
\hline & 1 & 2 & 3 & 4 & 5 & 6 & 7 & 8 & 9 & 10 \\
\hline MRS (control) & - & - & + & + & +++ & - & + & - & +++ & +++ \\
\hline MRS + erythritol & - & - & + & + & ++ & + & ++ & + & ++ & +++ \\
\hline MRS + lactitol & - & - & - & - & +++ & ++ & + & ++ & +++ & +++ \\
\hline MRS + xylitol & - & - & + & - & + & ++ & ++ & + & +++ & +++ \\
\hline MRS + maltitol & - & - & + & - & +++ & & +++ & ++ & ++ & +++ \\
\hline MRS + mannitol & - & - & + & - & +++ & +++ & ++ & + & +++ & +++ \\
\hline MRS + sorbitol & - & - & + & - & +++ & - & +++ & - & +++ & ++ \\
\hline MRS + gal-erythritol & ++ & + & $+/-$ & - & ++ & +++ & ++ & - & +++ & +++ \\
\hline MRS + gal-xylitol & - & $+/-$ & + & $+/-$ & +++ & +++ & +++ & $+/-$ & +++ & +++ \\
\hline MRS + gal-sorbitol & - & + & + & - & +++ & +++ & +++ & $+/-$ & $\mathrm{X}$ & +++ \\
\hline MRS + galactose & - & - & + & - & +++ & +++ & +++ & - & + & + \\
\hline MRS + galactose (glucose-free) & - & - & + & - & ++ & ++ & ++ & - & + & + \\
\hline
\end{tabular}

1. C. vini 0008, 2. C. vini 0009, 3. M. hiemalis, 4. G. candidum, 5. A. alternata, 6. A. brassicicola, 7. F. lateritium, 8. A. niger, 9. A. ochraceus, 10. Penicillium sp.

$X$ no tested, - no inhibition zone, $+/-$ inhibition zone between 0.5 and $2 \mathrm{~mm},+$ inhibition zone between 2.1 and $10 \mathrm{~mm},++$ inhibition zone between 10.1 and $20 \mathrm{~mm},+++$ inhibition zone above $20 \mathrm{~mm}, n t$ not tested 
Table 3 Concentration of polyols and saccharides before and after lactic acid fermentation by Lactobacillus pentosus ŁOCK 0979 in media containing polyols and their galactosyl derivatives

\begin{tabular}{|c|c|c|c|}
\hline Media & Compounds & Initial content $\left(\mathrm{g} \times \mathrm{L}^{-1}\right)$ & Residual content $\left(\mathrm{g} \times \mathrm{L}^{-1}\right)$ \\
\hline MRS (control) & Glucose & $20.9 \pm 3.61$ & - \\
\hline \multirow[t]{2}{*}{ MRS + erythritol } & Glucose & $25.4 \pm 0.01$ & - \\
\hline & Erythritol & $10.6 \pm 0.27 \mathrm{a}$ & $10.1 \pm 0.10 \mathrm{a}$ \\
\hline \multirow[t]{2}{*}{ MRS + lactitol } & Glucose & $20.2 \pm 0$ & - \\
\hline & Lactitol & $10.0 \pm 0 \mathrm{a}$ & $9.03 \pm 0.11 b$ \\
\hline \multirow[t]{2}{*}{ MRS + xylitol } & Glucose & $18.7 \pm 0.92$ & - \\
\hline & Xylitol & $10.3 \pm 0.10 \mathrm{a}$ & $10.0 \pm 0 \mathrm{a}$ \\
\hline \multirow[t]{2}{*}{ MRS + maltitol } & Glucose & $20.2 \pm 0.77$ & - \\
\hline & Maltitol & $10.4 \pm 0.54 \mathrm{a}$ & $10.0 \pm 0 \mathrm{a}$ \\
\hline \multirow[t]{2}{*}{ MRS + mannitol } & Glucose & $13.14 \pm 0.11$ & - \\
\hline & Mannitol & $10.0 \pm 0.04 \mathrm{a}$ & $7.4 \pm 0.70 b$ \\
\hline \multirow[t]{2}{*}{ MRS + sorbitol } & Glucose & $19.0 \pm 0.34$ & - \\
\hline & Sorbitol & $10.0 \pm 0 \mathrm{a}$ & $9.7 \pm 0.22 \mathrm{a}$ \\
\hline \multirow[t]{4}{*}{ MRS + gal-erythritol } & Glucose & $18.9 \pm 0.01$ & - \\
\hline & Gal-erythritol & $10.03 \pm 0 \mathrm{a}$ & $5.8 \pm 0.73 b$ \\
\hline & Erythritol & - & $1.7 \pm 0.09$ \\
\hline & Galactose & - & $0.6 \pm 0.09$ \\
\hline \multirow[t]{3}{*}{ MRS + gal-xylitol } & Glucose & $20.6 \pm 0$ & - \\
\hline & Gal-xylitol & $9.9 \pm 0.68 \mathrm{a}$ & $7.7 \pm 0.21 b$ \\
\hline & Xylitol & - & $1.0 \pm 0.07$ \\
\hline \multirow[t]{4}{*}{ MRS + gal-sorbitol } & Glucose & $18.7 \pm 0$ & - \\
\hline & Gal-sorbitol & $10.5 \pm 0.07 a$ & $7.8 \pm 0.38 b$ \\
\hline & Sorbitol & - & $0.7 \pm 0.08$ \\
\hline & Galactose & - & $0.1 \pm 0.02$ \\
\hline
\end{tabular}

Means designated with the same lowercase letter are not significantly different (Duncan's multiple range test)

- below the limit of detection polyols and gal-polyols was determined using HPLC, and the concentration of glucose was evaluated spectrophotometrically (Table 3). The results show that in lactic acid fermentation the bacteria used glucose as a primary carbon source, while the galactosyl-polyols and polyols (lactitol, mannitol) were used as additional carbon sources to varying degrees (Table 3).

The mannitol and lactitol present in the culture media were partially used by L. pentosus ŁOCK 0979 (as reflected in 10 and $26 \%$ decline in concentration after lactic acid fermentation, respectively). Galactosyl-polyols (gal-erythritol, gal-xylitol, and gal-sorbitol) were hydrolyzed to galactose and the respective polyols. Residual galactose was found in postfermentation CFS from samples supplemented with galerythritol and gal-sorbitol (Table 3). The content of erythritol, xylitol, maltitol, and sorbitol in the medium did not change significantly following lactic acid fermentation (Table 3).

\section{Acidity and Production of Acetic and Lactic Acids}

The concentration of acetic acid and lactic acid (L- and Denantiomers separately) was evaluated enzymatically using Megazyme kits. The total content of acetic acid was from
$4.09 \pm 0.178$ to $7.62 \pm 0.010 \mathrm{~g} \times \mathrm{L}^{-1}$, while that of lactic acid was from $4.58 \pm 0.390$ to $20.26 \pm 1.489 \mathrm{~g} \times \mathrm{L}^{-1}$; in all samples, the dominant stereoisomer was L-lactic acid accounting for $61-100 \%$ of the total (Table 4). The mean $\mathrm{pH}$ of the postfermentation supernatant was higher in the case of gal-polyols (pH $4.13 \pm 0.12$ ) than polyols ( $\mathrm{pH} 3.88 \pm 0.290$ ), except for sorbitol ( $\mathrm{pH}$ 4.4). Samples with higher $\mathrm{pH}$ (gal-polyols, sorbitol) exhibited a lower concentration of lactic acid

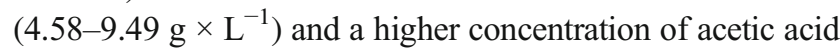
(5.76-7.62 $\left.\mathrm{g} \times \mathrm{L}^{-1}\right)$. L. pentosus ŁOCK 0979 generated the highest amounts of lactic and acetic acids in the presence of

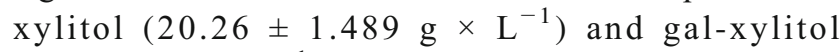
$\left(7.62 \pm 0.010 \mathrm{~g} \times \mathrm{L}^{-1}\right)$, respectively (Table 4$)$.

\section{Effects of Polyols and Gal-Polyols on the Content of Antifungal Acids}

The following 13 antifungal acids were quantified: DL-3phenyllactic acid (PLA), DL-p-hydroxyphenyllactic acid (HPLA), benzoic acid (BA), hydrocaffeic acid (HCaA), hydrocinnamic acid (HCiA), vanillic acid (VA), 4hydroxybenzoic acid (4-HBA), catechol (Cat), caffeic acid 
Table 4 Production of acetic, Dlactic, and L-lactic acids by Lactobacillus pentosus ŁOCK 0979 in the presence of polyols and gal-polyols

\begin{tabular}{|c|c|c|c|c|c|}
\hline \multirow[t]{2}{*}{ Medium } & \multirow[t]{2}{*}{$\mathrm{pH}$} & \multicolumn{3}{|c|}{ Production of lactic acid } & \multirow{2}{*}{$\begin{array}{l}\text { Production of acetic } \\
\text { acid }\left(g \times \mathrm{L}^{-1}\right)\end{array}$} \\
\hline & & $\begin{array}{l}\text { DL-Lactic acid } \\
\left(\mathrm{g} \times \mathrm{L}^{-1}\right)\end{array}$ & $\begin{array}{l}\% \text { D-lactic } \\
\text { acid }[\%]\end{array}$ & $\begin{array}{l}\% \text { L-lactic } \\
\text { acid [\%] }\end{array}$ & \\
\hline $\begin{array}{l}\text { Non-inoculated medium } \\
\text { MRS }\end{array}$ & $5.7 \pm 0.2$ & - & - & - & $0.07 \pm 0.000$ \\
\hline MRS (control) & 3.6 & $13.89 \pm 0.448$ & 7.0 & 93.0 & $4.39 \pm 0.105$ \\
\hline MRS + erythritol & 3.7 & $12.77 \pm 0.015$ & 0.0 & 100.0 & $4.75 \pm 0.178$ \\
\hline MRS + lactitol & 3.7 & $15.01 \pm 3.458$ & 6.6 & 93.3 & $4.09 \pm 0.178^{\mathrm{a}}$ \\
\hline MRS + xylitol & 4.0 & $20.26 \pm 1.489$ & 14.2 & 85.8 & $5.71 \pm 0.024^{\mathrm{a}}$ \\
\hline MRS + maltitol & 3.9 & $10.89 \pm 0.000$ & 0.0 & 100.0 & $4.87 \pm 0.003$ \\
\hline MRS + mannitol & 3.6 & $15.26 \pm 1.619$ & 5.1 & 94.9 & $5.76 \pm 0.020^{\mathrm{a}}$ \\
\hline MRS + sorbitol & 4.4 & $5.74 \pm 1.257$ & 34.8 & 65,2 & $6.52 \pm 0.010^{\mathrm{a}}$ \\
\hline MRS + gal-erythritol & 4.2 & $9.49 \pm 0.234$ & 37.7 & 62.3 & $6.61 \pm 0.023^{\mathrm{a}}$ \\
\hline MRS + gal-xylitol & 4.2 & $8.81 \pm 1.685$ & 38.6 & 61.4 & $7.62 \pm 0.010^{\mathrm{a}}$ \\
\hline MRS + gal-sorbitol & 4.0 & $4.58 \pm 0.390$ & 31.9 & 68.1 & $7.17 \pm 0.020^{\mathrm{a}}$ \\
\hline
\end{tabular}

${ }^{a}$ Significantly different from the control test
(CaA), ferulic acid (FA), 3-hydroxybenzoic acid (3-HBA), 2,4-dihydroxybenzoic acid (2,4-dHBA), and p-coumaric acid (p-CoumA), using a UHPLC-MS system coupled with QuEChERS. It was found that the minimum inhibitory concentrations of the tested acids are many times higher than their actual concentrations in CFS (Table 5). Statistically significant differences in the content of PLA and HCaA were linked to the composition of the culture medium. PLA content in CFS from the bacterial culture with gal-xylitol differed from that found in cultures with lactitol and xylitol. In the presence of galerythritol and gal-xylitol, L. pentosus ŁOCK 0979 produced double to triple the amount of vanillic acid and half the amount of Cat as compared to the other CFS. The content of HCaA, HCiA, VA, 4-HBA, Cat, CaA, FA, 3-HBA, 2,4dHBA, and p-CoumA was low and ranged from approx. 0 $(<\mathrm{LOD})$ to $0.161 \mathrm{mg} \times \mathrm{L}^{-1}$ (Table 5).

\section{Production of Hydroxy Fatty Acids in the Presence of Mannitol}

Volatile compounds with potential antifungal properties (fatty acids, hydroxy fatty acids) synthesized by L. pentosus ŁOCK 0979 cultured in MRS and in the presence of $1 \%(\mathrm{~m} / \mathrm{v})$ mannitol were identified (Table 6). Mannitol induced antagonistic activity of L. pentosus LOCK 0979 against some of the test fungi (A. brassicicola, A. niger, F. lateritium), which must therefore be attributable to one or more metabolites synthesized in the presence of this polyol. Moreover, CFS samples revealed 2-hydroxy-4-methylpentanoic acid, a compound described by Ndagano et al. [19] as a strong antifungal agent.

\section{Enzymatic Activity of Lactobacillus pentosus LOCK 0979}

Examination of the activity of enzymes metabolizing lipids, proteins, and phosphates revealed some minor differences between L. pentosus ŁOCK 0979 cultures conducted in media supplemented with various polyols and gal-polyols (Table 7). As compared to the controls, esterase activity was found only in the presence of lactitol and gal-sorbitol, while that of esterase lipase in the presence of gal-erythritol.

\section{Effects of Polyols and Gal-Polyols on Fungal Growth and Morphology}

The yeasts $C$. vini ŁOCK 0008 and ŁOCK 0009 and the mold A. brassicicola were examined microscopically following culture in Sabouraud agar with 10\% $(v / v)$ CFS. The results are given in Tables 8 and 10. Scanning electron micrographs of C. vini LOCK 0009 cultivated in the presence of CFSs are presented in Table 9.

Light microscopy revealed greater cell differentiation in the yeast $C$. vini ŁOCK 0008 grown in Sabouraud agar with $10 \%$ $(v / v)$ CFS from L. pentosus ŁOCK 0979 cultured in the presence of gal-erythritol than the control (Table 8). Candida vini ŁOCK 0008 cells were at different developmental stages and included both single cells and some initial degrees of pseudomycelium formation. The same was true of $C$. vini ŁOCK 0009 grown in Sabouraud agar with 10\% (v/v) CFS from $L$. pentosus ŁOCK 0979 cultured in the presence of galpolyols (gal-erythritol, gal-xylitol, gal-sorbitol). What is more, the addition of gal-polyols to the bacterial medium led to fungal deformation and gave rise to blastoconidia (CFS galerythritol, CFS gal-xylitol, CFS gal-sorbitol) (red arrows in the Table 8). The yeast cells were narrower, and some of them 
Table 5 Content of antifungal acids produced by Lactobacillus pentosus ŁOCK 0979 as determined by HPLC coupled with QuEChERS

\begin{tabular}{|c|c|c|c|c|c|c|}
\hline Polyols in medium & \multicolumn{6}{|c|}{ Production of antifungal acids and their referential MIC $\left(\mathrm{mg} \times \mathrm{L}^{-1}\right)$} \\
\hline & PLA & & HPLA & $\mathrm{BA}^{*}$ & 3-HBA* & $\mathrm{HCaA}$ \\
\hline MRS-non-inoculated & $0.107 \pm 0.001$ & & $0.033 \pm 0.003$ & $0.339 \pm 0.010$ & $0.062 \pm 0.002$ & $0.006 \pm 0.001$ \\
\hline MRS (control) & $41.513 \pm 1.175$ & & $4.255 \pm 0.069$ & $0.623 \pm 0.049$ & $0.072 \pm 0.002$ & $0.090 \pm 0.001$ \\
\hline Erythritol & $40.319 \pm 0.865$ & & $4.132 \pm 0.045$ & $0.614 \pm 0.103$ & 0.072 .002 & $0.062 \pm 0$ \\
\hline Lactitol & $51.228 \pm 0.482 \mathrm{x}$ & & $4.872 \pm 0.161$ & $0.504 \pm 0.022$ & $0.078 \pm 0.001$ & $0.088 \pm 0.001$ \\
\hline Xylitol & $49.988 \pm 1.026 z$ & & $4.869 \pm 0.052$ & $0.504 \pm 0.017$ & $0.079 \pm 0.001$ & $0.083 \pm 0.003$ \\
\hline Maltitol & $24.224 \pm 16.272$ & & $2.844 \pm 1405$ & $0.249 \pm 0.094$ & $0.050 \pm 0.023$ & $0.060 \pm 0.033$ \\
\hline Mannitol & $40.992 \pm 0.875$ & & $4.128 \pm 0.096$ & $0.582 \pm 0.007$ & $0.073 \pm 0.001$ & $0.085 \pm 0.003$ \\
\hline Sorbitol & $31.962 \pm 14.709$ & $3.93=$ & & 0.282 & $0.06 \pm 0.017$ & $0.06 \pm 0.032$ \\
\hline Gal-erythritol & $21.91 \pm 5.455$ & $6.13=$ & & 0.010 & $0.06 \pm 0.013$ & $0.04 \pm 0.010$ \\
\hline Gal-xylitol & $16.65 \pm 1.679 \mathrm{X}, \mathrm{Z}$ & $2.46=$ & & 0.005 & $0.05 \pm 0.005$ & $0.03 \pm 0.003 X$ \\
\hline Gal-sorbitol & $24.89 \pm 8.188$ & 3.14 & & 0.106 & $0.06 \pm 0$ & $0.04 \pm 0.018$ \\
\hline MIC (Oliveira et al. 2015) & $7500-10,000$ & - & & & - & $>10,000$ \\
\hline Polyols in medium & Production of antifun & gal acids and tl & eferential MIC & $\left.\times L^{-1}\right)$ & & \\
\hline & HPLA & 2,4-dHBA & Cat & 4-HBA & Vanillic acid & $\mathrm{HCiA}$ \\
\hline MRS - non-inoculated & $0.033 \pm 0.003$ & $0.031 \pm 0.001$ & $0.015 \pm 0.002$ & $<0.008$ & $0.186 \pm 0.006$ & $<0.017$ \\
\hline MRS (control) & $4.255 \pm 0.069$ & $0.012 \pm 0.002$ & $0.045 \pm 0.003$ & $<0.008$ & $0.003 \pm 0$ & $<0.017$ \\
\hline Erythritol & $4.132 \pm 0.045$ & $0.010 \pm 0$ & $0.037 \pm 0$ & $<0.008$ & $0.086 \pm 0.007$ & $<0.017$ \\
\hline Lactitol & $4.872 \pm 0.161$ & $0.011 \pm 0.001$ & $0.048 \pm 0.004$ & $<0.008$ & $0.070 \pm 0.003$ & $<0.017$ \\
\hline Xylitol & $4.869 \pm 0.052$ & $0.011 \pm 0.001$ & $0.039 \pm 0.003$ & $<0.008$ & $0.075 \pm 0.007$ & $<0.017$ \\
\hline Maltitol & $2.844 \pm 1405$ & $0.009 \pm 0.002$ & $0.034 \pm 0.007$ & $<0.008$ & $0.041 \pm 0.015$ & $<0.017$ \\
\hline Mannitol & $4.128 \pm 0.096$ & $0.011 \pm 0.001$ & $0.049 \pm 0.008$ & $<0.008$ & $0.086 \pm 0.003$ & $<0.017$ \\
\hline Sorbitol & $3.93 \pm 0.852$ & $0.01 \pm 0.002$ & $0.040 \pm 0.003$ & $<0.008$ & $0.084 \pm 0.012$ & $<0.017$ \\
\hline Gal-erythritol & $6.13 \pm 4.311$ & $0.02 \pm 0.001$ & $0.018 \pm 0.005$ & $<0.008$ & $0.161 \pm 0.057$ & $<0.017$ \\
\hline Gal-xylitol & $2.46 \pm 0.217$ & $0.02 \pm 0.001$ & $0.017 \pm 0.001$ & $0.012 \pm 0.001$ & $0.127 \pm 0.001$ & $<0.017$ \\
\hline Gal-sorbitol & $3.14 \pm 0.753$ & $0.01 \pm 0.005$ & $0.044 \pm 0.001$ & $<0.008$ & $0.005 \pm 0$ & $<0.017$ \\
\hline MIC (Oliveira et al. 2015) & - & - & $>1000$ & $>1000$ & $>100$ & $100-1000$ \\
\hline
\end{tabular}

Distributions with both a capital and small letter are significantly different from each other (Tukey's test $p \leq 0.05$ )

PLA DL-3-phenyllactic acid, HPLA DL-p-hydroxyphenyllactic acid, $B A$ benzoic acid, 3-HBA 3-hydroxybenzoic acid, $H C a A$ hydrocaffeic acid, 2,4- $d H B A$ 2,4-dihydroxybenzoic acid, Cat catechol, 4-HBA 4-hydroxybenzoic acid, VA vanillic acid, $C a A$ caffeic acid, $p$-CoumA p-coumaric acid, FA ferulic acid, HCiA hydrocinnamic acid

$* p$ value estimated in Tukey's test $\leq 0.05$ (significance of differences may not be estimated)

became pear-shaped as compared to the controls (Table 8). SEM provided more details about the form and surface of C. vini ŁOCK 0009. In the control, yeasts were elliptical and developed pseudohyphae with smooth and flat surfaces (Table 9). The cells of $C$. vini ŁOCK 0009 cultivated with the CFS of L. pentosus ŁOCK 0979 in the presence of galpolyols were strongly deformed. Their shape was warped and the surface rough, and cell damage was visible in the form of concave areas on the surface (Table 9). Additionally, in the presence of CFS gal-xylitol yeasts, cells were coated by extracellular matrix (Table 9).

Morphological changes were also observed in the mycelium of the mold A. brassicicola grown in Sabouraud agar with $10 \%(v / v)$ CFS from L. pentosus ŁOCK 0979 cultured in bacterial media supplemented with erythritol and xylitol. Furthermore, growth inhibition and mycelium deformation were found for all gal-polyols in the bacterial medium (Table 10). The addition of lactitol and mannitol to the bacterial medium led to complete inhibition of fungal growth (hence no photomicrographs).

\section{Discussion}

The control of spoilage microorganisms and, by the same token, the extension of the shelf-life of foodstuffs still pose formidable challenges. While the use of Lactobacillus sp. as natural bioprotective agents was already reported by Magnusson [14], a definitive explanation of their mechanism of action against undesirable fungi was not provided.

Lactobacilli inhibit the growth of other bacteria (of the same or different species) as well as that of fungi, including 
Table 6 Volatile compounds produced by Lactobacillus pentosus ŁOCK 0979 in MRS broth and in the presence of mannitol

\begin{tabular}{|c|c|c|c|c|}
\hline \multirow{2}{*}{\multicolumn{2}{|c|}{$\begin{array}{l}\text { No. } \\
\text { Compound }\end{array}$}} & \multicolumn{3}{|l|}{ Media } \\
\hline & & \multirow{2}{*}{$\begin{array}{l}\text { MRS - non-inoculated } \\
+\end{array}$} & \multirow{2}{*}{$\begin{array}{l}\text { MRS (control) } \\
+\end{array}$} & \multirow{2}{*}{$\begin{array}{l}\text { MRS + mannitol } \\
+\end{array}$} \\
\hline 1 & Butanoic acid & & & \\
\hline 2 & 2-Methylbutanoic acid & + & + & - \\
\hline 3 & Isovaleric acid & + & + & + \\
\hline 4 & Caproic acid & + & + & + \\
\hline 5 & 3-Hydroxypropanoic acid & - & + & + \\
\hline 7 & 2-Hydroxypropanoic acid & + & + & + \\
\hline 8 & 2-Hydroxyisocaproic acid & - & + & + \\
\hline 9 & Octanoic acid & + & + & + \\
\hline 10 & 2-Hydroxy-3-methylbutyric acid & - & + & + \\
\hline 11 & 2-Hydroxybutanoic acid & + & + & - \\
\hline 12 & 2-Methylthioacetic acid & + & + & - \\
\hline 13 & Acetic acid & + & + & - \\
\hline 14 & Acetoxyacetic acid & + & - & - \\
\hline 17 & 2-Hydroxy-3-methylpentanoic acid & - & + & - \\
\hline 18 & 2-Hydroxy-4-methylpentanoic acid & - & + & + \\
\hline 19 & 3-(Methylthio)propanoic acid & - & + & - \\
\hline 20 & 3-Hydroxypropanoic acid & - & + & - \\
\hline 21 & Decanoic acid & + & - & - \\
\hline 22 & Butanedioic acid & + & - & + \\
\hline 23 & 2-Oxopentanedioic acid & - & + & - \\
\hline 24 & Benzoic acid & + & + & + \\
\hline 25 & 2-Hydroxypropanoic acid & + & - & + \\
\hline 27 & Phenylacetic acid & + & + & + \\
\hline 28 & Dodecanoic acid & - & + & + \\
\hline 29 & Hydrocinnamic acid & - & + & - \\
\hline 30 & Myristic acid & + & + & + \\
\hline 32 & Pentadecanoic acid & - & + & - \\
\hline 33 & Azelaic acid, dimethyl ester & + & + & - \\
\hline 34 & Palmitic acid & + & + & + \\
\hline 35 & 2-Pyrrolidone-5-carboxylic acid & - & - & + \\
\hline 36 & Palmitoleic acid & + & + & + \\
\hline 37 & 2-Hydroxybenzenepropanoic acid & - & + & + \\
\hline 38 & Heptadecanoic acid (C17:0) & + & + & - \\
\hline 39 & Citric acid, trimethyl ester & + & - & - \\
\hline 40 & Methyl stearate & + & + & + \\
\hline 37 & 9-Octadecenoic acid & + & + & + \\
\hline 38 & 11-Octadecenoic acid & - & + & - \\
\hline 39 & Linoleic acid & - & + & + \\
\hline 40 & 9,12-Octadecadienoic acid & + & - & - \\
\hline 37 & Linolelaidic acid & - & - & + \\
\hline 38 & 9,12-Octadecadienoic acid & + & + & + \\
\hline 39 & Eicosanoic acid & + & + & - \\
\hline 40 & 11-Eicosenoic acid & - & - & + \\
\hline 41 & 3-Octyloxiraneoctanoic acid & + & + & + \\
\hline 42 & 10-hydroxyoctadecanoic acid & - & + & - \\
\hline
\end{tabular}







Table 8. Micrographs of yeasts grown for 2 days in Sabouraud medium with $10 \%$ ( $v / v$ ) cell-free supernatant of Lactobacillus pentosus ŁOCK 0979 cultivated $48 \mathrm{~h}$ in the media containing gal-polyols

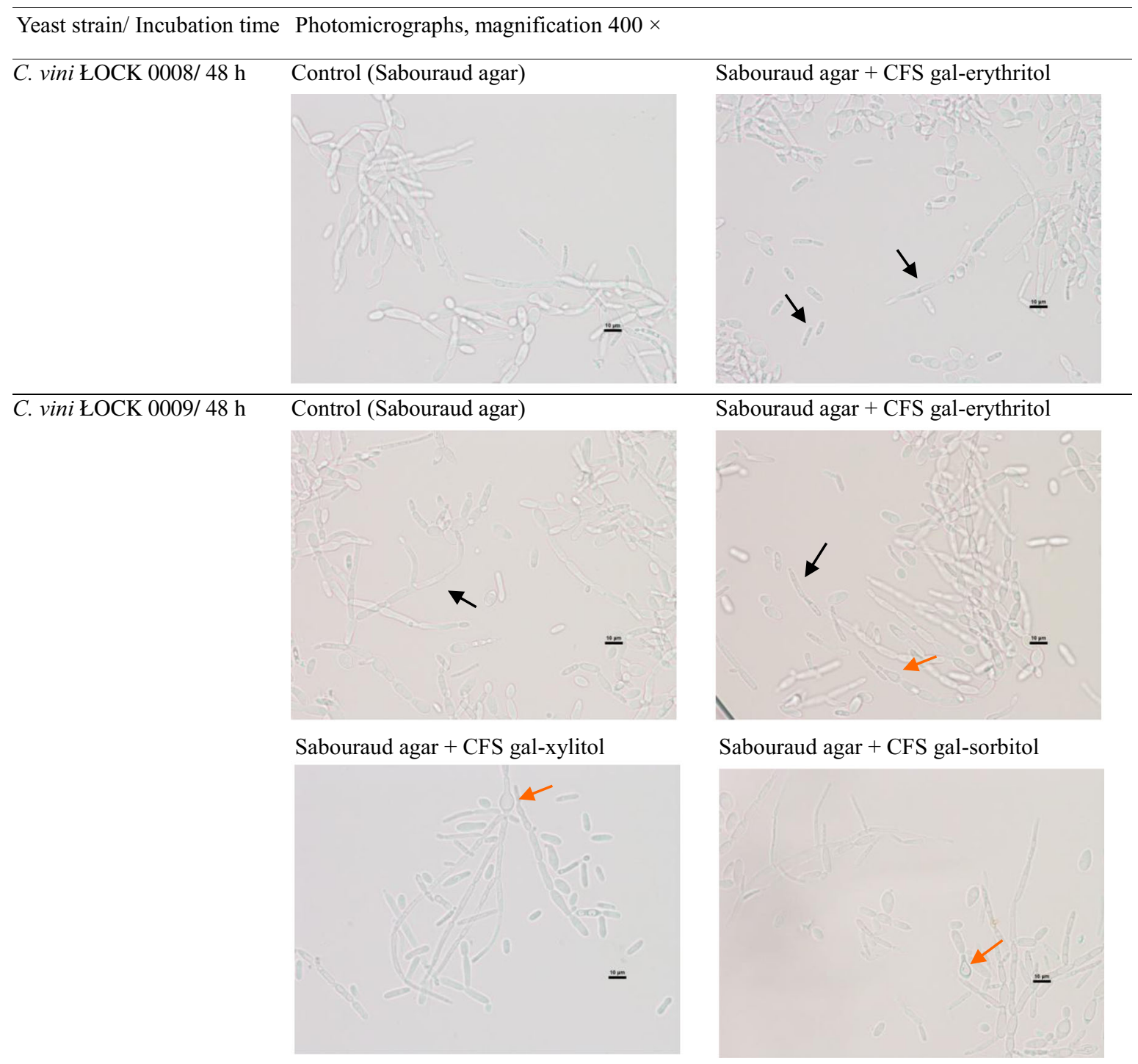

pathogenic and toxin-producing molds [1, 7]. While many authors have reported the antifungal properties of LAB [3, 10], their exact underlying mechanisms remain elusive. Nevertheless, it is known that a major role is played by some bacterial metabolites, and especially by organic acids, hydroxy fatty acids, cyclic dipeptides, and low molecular weight proteinaceous compounds [22]. In addition to primary metabolites (lactic and acetic acids), which are produced by all Lactobacilli. sp., some LAB species synthesize secondary metabolites, which may selectively affect other microorganisms; these include propionic, hexanoic, salicylic, succinic, formic, 2-pyrrolidone-5-carboxylic, 3-phenyllactic, and 4-hydroxyphenyllactic acids $[2,14,17]$. One of the best described antifungal products of lactic acid fermentation is 3-phenyllactic acid (PLA), synthesized by LAB such as L. casei, L. fermentum, $L$. rhamnosus, $L$. reuteri, and $L$. sakei $[14,17,18]$. While L. pentosus ŁOCK 0979 does produce PLA, its concentration in the CFS is much lower than its minimum inhibitory concentration reported by other authors [20].

The antifungal metabolites of Lactobacillus sp. constitute a rich mixture of active compounds whose qualitative and quantitative composition largely depends on the compounds found in the bacterial culture medium. Ndagano et al. [19], who evaluated the effects of different concentrations and proportions of acetic and lactic acids on fungal viability, observed significant synergies: the mixture was more potent than the sum of its individual components taken together. Synergies 
Table 9. Scanning electron micrographs of yeasts grown for 2 days on Sabouraud agar medium with $10 \%(v / v)$ CFS from $48 \mathrm{~h}$ culture of Lactobacillus pentosus ŁOCK 0979 in media containing gal-polyols



Control medium (Sabouraud agar)

Sabouraud agar + CFS gal-xylitol

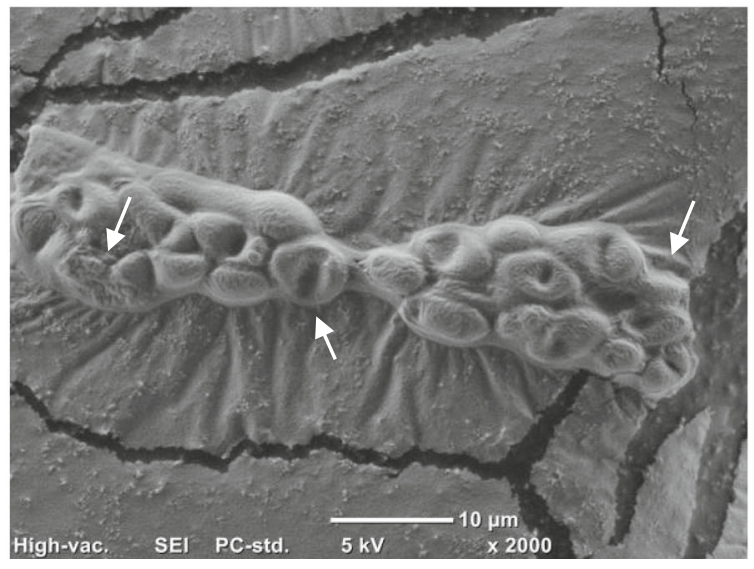

\section{Sabouraud agar + CFS gal-erythritol}
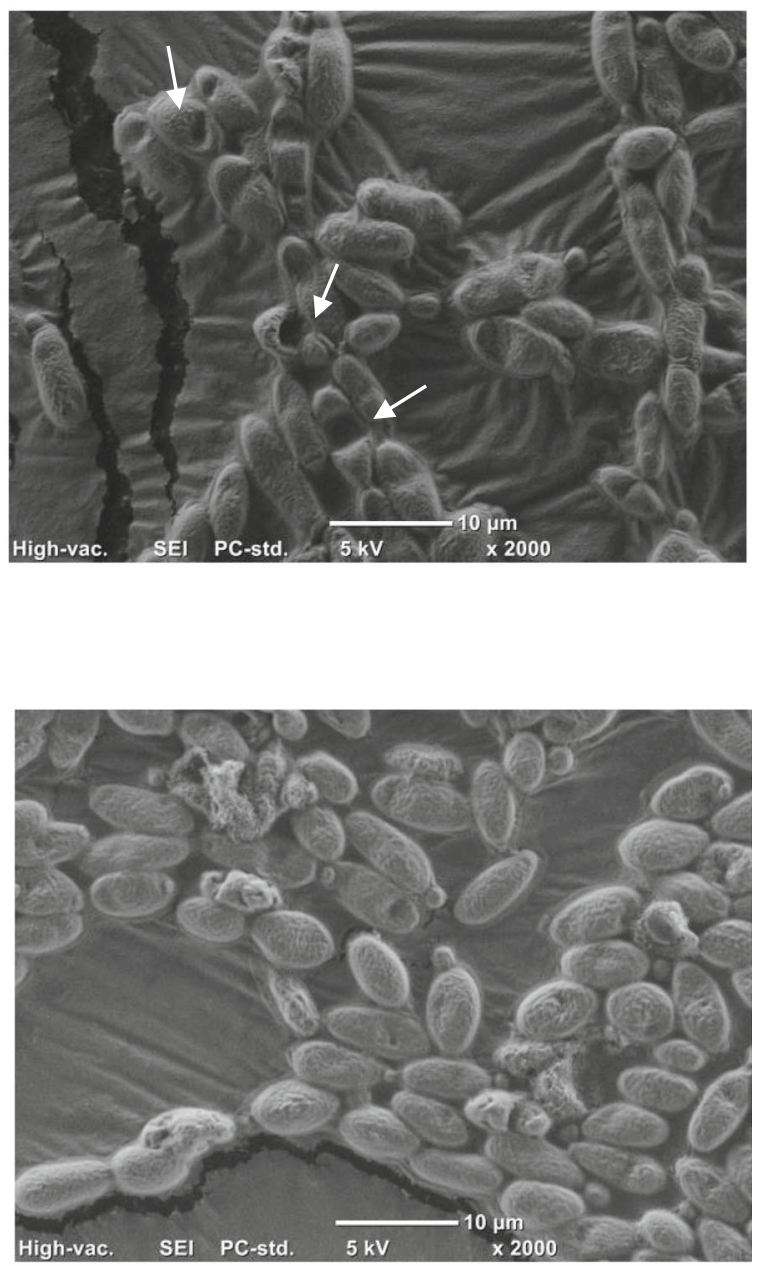

Sabouraud agar + CFS gal-sorbitol may also be found for some parameters of the culture medium, such as $\mathrm{pH}$.

The study presented herein was preceded by evaluation of 60 Lactobacillus sp. strains, including L. pentosus ŁOCK 0979, cultured in the presence of polyols and galactosyl-polyols as alternative carbon sources [13] to select bacteria with strong antagonistic properties against as many indicator fungal strains as possible. L. pentosus ŁOCK 0979 was selected for further research as one of the most prospective antifungal strain. In this context, the use of polyols and their galactosyl derivatives to enhance the inhibitory properties of lactobacilli is a novel solution which offers a promising method for modulating LAB metabolism. In situ studies on food products describing enhanced antifungal activity of lactobacilli on fruits have been presented by Lipinska et al. [13].

In the presented experiments, L. pentosus LOCK 0979 exhibited the ability to partially absorb lactitol, mannitol, and all the tested galactosyl-polyols. While Tyler et al. [30] isolated Lactobacillus florum 2F, a heterofermentative strain which can biosynthesize erythritol and mannitol, the consumption of polyols and galactosyl-polyols by Lactobacillus bacteria represents a new line of research with scant available literature.

Since the antifungal activity of lactobacilli consists of a complex set of interactions beginning at the cellular level, in this study considerable attention was given to the enzymatic activity of the bacteria in the presence of polyols 
Table 10. Morphological changes in the mycelia of Alternaria brassicicola grown for 7 days in Sabouraud medium with $10 \%$ ( $v / v)$ CFS from $48 \mathrm{~h}$ cultures of L. pentosus ŁOCK 0979 in media containing polyols and gal-polyols

Photomicrographs of the morphological changes in the mycelia of Alternaria brassicicola, (magnification $400 \times$ )

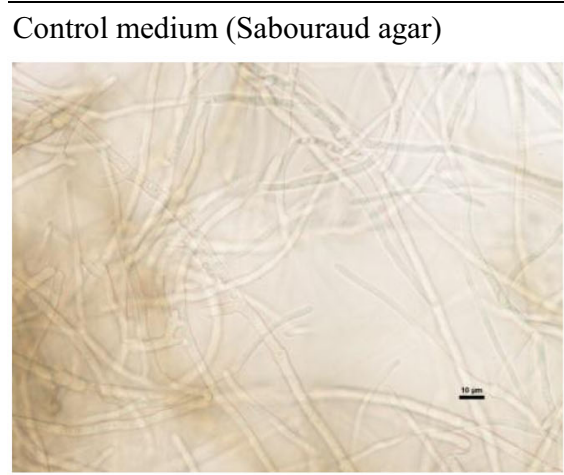

Sabouraud agar + CFS gal-erythritol

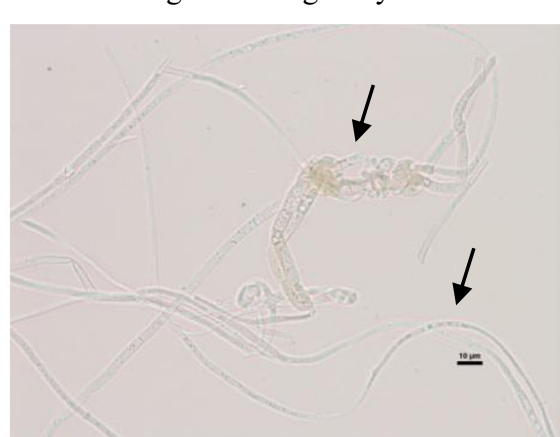



Sabouraud agar + CFS gal-xylitol

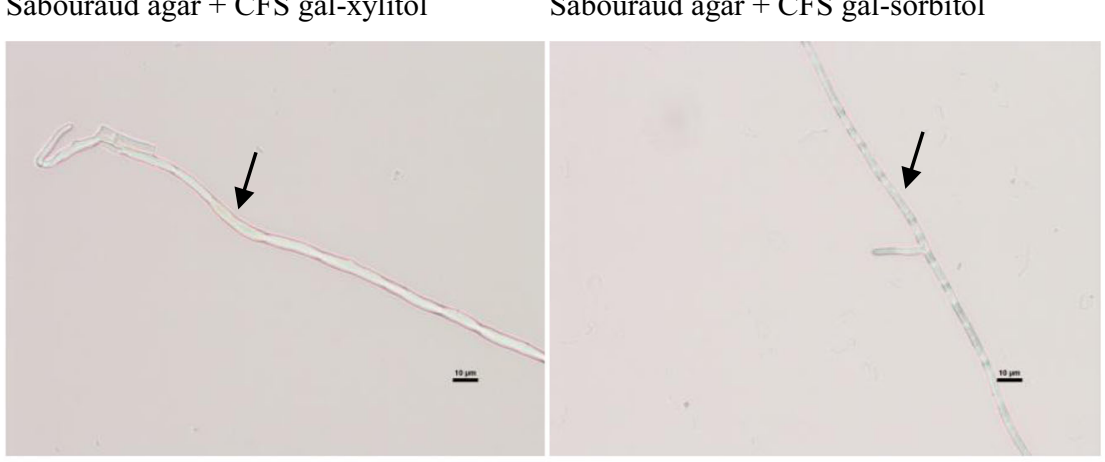

Sabouraud agar + CFS xylitol

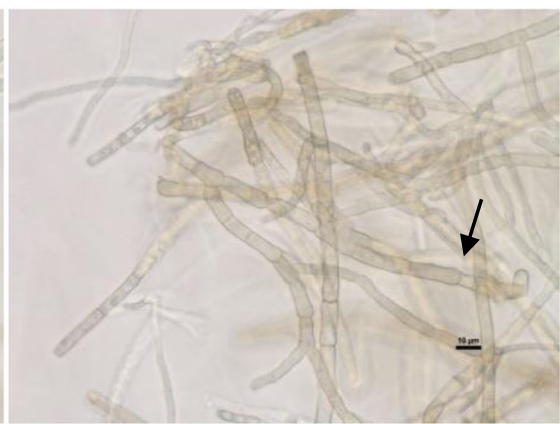

Sabouraud agar + CFS gal-sorbitol and gal-polyols. Some small differences were found in enzymes metabolizing lipids, proteins, and phosphates, and in particular in esterase and esterase lipase, which catalyze the hydrolysis and synthesis of organic acid esters, primarily from water-soluble substrates, such as triacylglycerols containing short chain fatty acids. Bacterial esterase activity promotes the hydrolysis of a wide spectrum of substrates to acids [5, 28], including antifungal metabolites. LAB enzymes can be used to modify the gustatory and olfactory properties of wines and cheeses and to produce some ingredients of foodstuffs, pharmaceuticals, and cosmetics [12]. Fatty acids and hydroxy fatty acids synthesized by LAB affect fungal viability by irreversibly weakening and deforming the lipid bilayer [23]. The morphological changes in the structure of the cell walls of $C$. vini (strains 0008 and 0009) and A. brassicicola mycelia, which were revealed in this study using SEM and light microscopy, corroborate that mechanism of action for the obtained CFSs, which led to strong deformation of yeasts' surface; similar morphological changes of Candida sp. were described by Shengli et al. [27]. In the presence of CFSs, yeasts can produce extracellular matrix to promote their adherence and protect cells from environmental insults [29].

\section{Conclusions}

The antifungal activity of L. pentosus ŁOCK 0979 depends on the bacterial culture medium as well as on the fungal strain. The present study shows changes in the antifungal profile of the studied bacterial strain linked to the composition of the culture medium. Although no single decisive factor (metabolite) was found to be responsible for inhibiting fungal growth, the results indicate how bacterial metabolite profiles may be beneficially modulated. Thus, the authors have broken new ground in developing natural ways of ensuring the microbiological safety of food.

Acknowledgements The authors thank Jaroslaw Arkusinski for technical assistance.

Funding This study was funded by the National Science Center (grant no. 2013/09/B/NZ9/01806).

\section{Compliance with Ethical Standards}

Ethical Statement All authors of this paper have read and approved the final version submitted. The contents of this manuscript have not been copyrighted or published previously.

1. The contents of this manuscript are not now under consideration for publication elsewhere. 
2. The contents of this manuscript will not be copyrighted, submitted, or published elsewhere, while acceptance by the Journal is under consideration.

3. All procedures performed in this studies have not been conducted in human participants and/or animals.

Conflict of Interest The authors declare that they have no conflict of interest.

Open Access This article is distributed under the terms of the Creative Commons Attribution 4.0 International License (http:// creativecommons.org/licenses/by/4.0/), which permits unrestricted use, distribution, and reproduction in any medium, provided you give appropriate credit to the original author(s) and the source, provide a link to the Creative Commons license, and indicate if changes were made.

\section{References}

1. Arasu MV, Al-Dhabi NA, Rejiniemon TS, Lee KD, Huxley VAJ, Kim DH, Duraipandiyan V, Karuppiah P, Choi KC (2015) Identification and characterization of Lactobacillus brevis $\mathrm{P} 68$ with antifungal, antioxidant and probiotic functional properties. Indian J Microbiol 55:19-28. https://doi.org/10.1007/s12088-014-0495-3

2. Belguesmia $Y$, Rabesona H, Mounier J, Pawtowsky A, Le Blay G, Barbier G, Haertlé T, Chobert JM (2014) Characterization of antifungal organic acids produced by Lactobacillus harbinensis K.V9.3.1Np immobilized in gellan-xanthan beads during batch fermentation. Food Control 36:205-211. https://doi.org/10.1016/j. foodcont.2013.08.028

3. Crowley S, Mahony J, van Sinderen D (2013) Current perspectives on antifungal lactic acid bacteria as natural bio-preservatives. Trends Food Sci Technol 33:93-109. https://doi.org/10.1016/j.tifs. 2013.07.004

4. Delavenne E, Cliquet S, Trunet C, Barbier G, Mounier J, Le Blay G (2015) Characterization of the antifungal activity of Lactobacillus harbinensis K.V9.3.1 Np and Lactobacillus rhamnosus K.C8.3.1I in yogurt. Food Microbiol 45:10-17. https://doi.org/10.1016/j.fm. 2014.04.017

5. Esteban-Torres M, Reverón I, Santamaría L, Mancheño JM, de las Rivas B, Muñoz R (2016) The Lp_3561 and Lp_3562 enzymes support a functional divergence process in the lipase/esterase toolkit from Lactobacillus plantarum. Front Microbiol 7:1118. https://doi. org/10.3389/fmicb.2016.01118

6. FAO (2013) Food wastage footprint, Impacts on natural resources, Summary report, Rzym, http://www.fao.org/docrep/018/i3347e/ i3347e.pdf Accessed 29 Aug 2017

7. Goderska K, Rychlik T, Andrzejewska E, Szkaradkiewicz A, Czarnecki Z (2012) Antagonistyczny wpływ Lactobacillus acidophilus DSM 20079 i DSM 20242 na bakterie patogenne izolowane od ludzi [Antagonistic impact of Lactobacillus acidophilus DSM 20079 and DSM 20242 strains on pathogenic bacteria isolated from people]. NTJ 82:114-131. http://www.pttz.org/zyw/ wyd/czas/2012,\%203(82)/10 Goderska.pdf Accessed 29 Aug 2017

8. Irazoqui G, Giacomini C, Batista-Viera F, Brena BM, CardelleCobas A, Corzo N, Jimeno ML (2009) Characterization of galactosyl derivatives obtained by transgalactosylation of lactose and different polyols using immobilized $\beta$-galactosidase from Aspergillus oryzae. J Agric Food Chem 57:11302-11307. https:// doi.org/10.1021/jf901834k

9. Klewicka E (2007) Antifungal activity of lactic acid bacteria of genus Lactobacillus sp. in the presence of polyols. Acta Aliment 36:495-499. https://doi.org/10.1556/AAlim.2007.0004

10. Klewicka E, Lipińska L (2016) Aktywność przeciwgrzybowa bakterii fermentacji mlekowej z rodzaju Lactobacillus [Antifungal activity of lactic acid bacteria]. ŻNTJ 104:17-31. https://doi.org/10. 15193/zntj/2016/104/098

11. Klewicki R (2007) Effect of selected parameters of lactose hydrolysis in the presence of $\beta$-galactosidase from various sources on the synthesis of galactosyl-polyol derivatives. Eng Life Sci 7:268-274. https://doi.org/10.1002/elsc.200620185

12. Lewandowska M, Bednarski W, Wachowska M, Kordala N (2015) Charakterystyka, właściwości oraz znaczenie biotechnologiczne esteraz bakteryjnych [Characteristic, properties and biological impact of bacterial esterases]. Adv. Agric Sci 583:85-96 http://agro. icm.edu.pl/agro/element/bwmeta1.element.agro-129a4c34-4a33442c-b238-6c39558743d2 Accessed 29 Aug 2017

13. Lipińska L, Klewicki R, Klewicka E, Kołodziejczyk K, Sójka M, Nowak A (2016) Antifungal activity of lactobacillus sp. bacteria in the presence of xylitol and galactosyl-xylitol. Biomed Res Int 2016. https://doi.org/10.1155/2016/5897486

14. Magnusson J (2003) Antifungal activity of lactic acid bacteria. $\mathrm{PhD}$ thesis. Swedish University of Agricultural Sciences, Uppsala

15. Magnusson J, Schnurer J (2001) Lactobacillus coryniformis subsp. coryniformis strain Si3 produces a broad-spectrum proteinaceous antifungal compound. Appl Environ Microbiol 67:1-5. https://doi. org/10.1128/AEM.67.1.1-5.2001

16. Magnusson J, Ström K, Roos S, Sjögren J, Schnürer J (2003) Broad and complex antifungal activity among environmental isolates of lactic acid bacteria. FEMS Microbiol Lett 219:129-135. https://doi. org/10.1016/S0378-1097(02)01207-7

17. Mu W, Yang Y, Jia J, Zhang T, Jiang B (2010) Production of 4hydroxyphenyllactic acid by Lactobacillus sp. SK007 fermentation. J Biosci Bioeng 109:369-371. https://doi.org/10.1016/j.jbiosc. 2009.10.005

18. Mu W, Yu S, Zhu L, Zhang T, Jiang B (2012) Recent research on 3phenyllactic acid, a broad-spectrum antimicrobial compound. Appl Microbiol Biotechnol 95:1155-1163. https://doi.org/10.1007/ s00253-012-4269-8

19. Ndagano D, Lamoureux T, Dortu C, Vandermoten S, Thonart $\mathrm{P}$ (2011) Antifungal activity of 2 lactic acid bacteria of the Weissella genus isolated from food. J Food Sci 76:M305-M311. https://doi.org/10.1111/j.1750-3841.2011.02257.x

20. Oliveira PM, Brosnan B, Furey A, Coffey A, Zannini E, Arendt EK (2015) Lactic acid bacteria bioprotection applied to the malting process. Part I: strain characterization and identification of antifungal compounds. Food Control 51:433-443. https://doi.org/10.1016/ j.foodcont.2014.07.004

21. Oliveira PM, Zannini E, Arendt EK (2014) Cereal fungal infection, mycotoxins, and lactic acid bacteria mediated bioprotection: from crop farming to cereal products. J Food Microbiol 37:78-95. https:// doi.org/10.1016/j.fm.2013.06.003

22. Pawlowska AM, Zannini E, Coffey A, Arendt EK (2012) "Green Preservatives": combating fungi in the food and feed industry by applying antifungal lactic acid bacteria. Adv Food Nutr Res 66: 217-238. https://doi.org/10.1016/B978-0-12-394597-6.00005-7

23. Pohl CH, Kock JL, Thibane VS (2011) Antifungal free fatty acids: a review. In: Science against microbial pathogens: current research 
and technological advances, Formatex Research Centre, Badajoz, Spain, pp 61-71

24. Russo P, Arena MP, Fiocco D, Capozzi V, Drider D, Spano G (2016) Lactobacillus plantarum with broad antifungal activity: a promising approach to increase safety and shelf-life of cerealbased products. Int J Food Microbiol 247:48-54. https://doi.org/ 10.1016/j.ijfoodmicro.2016.04.027

25. Ryan LA, Zannini E, Dal Bello F, Pawlowska A, Koehler P, Arendt EK (2011) Lactobacillus amylovorus DSM19280 as a novel foodgrade antifungal agent for bakery products. Int J Food Microbiol 146:276-283. https://doi.org/10.1016/j.ijfoodmicro.2011.02.036

26. Ryu EH, Yang EY, Woo ER, Chang HC (2014) Purification and characterization of antifungal compounds from Lactobacillus plantarum HD1 isolated from kim chi. Food Microbiol 41:19-26. https://doi.org/10.1016/j.fm.2014.01.011
27. Shengli M, Zhao Y, Xia X, Dong X, Ge W, Li H (2015). Effects of streptococcus sanguinis bacteriocin on cell surface hydrophobicity, membrane permeability, and ultrastructure of Candida thallus. BioMed Res Int 2017. https://doi.org/10.1155/2017/5291486

28. Song YR, Baik SH (2017) Molecular cloning, purification, and characterization of a novel thermostable cinnamoyl esterase from Lactobacillus helveticus KCCM 11223. Prep Biochem Biotechnol 47:496-504. https://doi.org/10.1080/10826068.2016.1275011

29. Taff HT, Mitchell KF, Edward JA, Andes DR (2013) Mechanisms of Candida biofilm drug resistance. Future Microbiol 8:1325-1337. https://doi.org/10.2217/fmb.13.101

30. Tyler C, Kopit L, Doyle C, AO Y, Hugenholtz J, Marco ML (2016) Polyol production during heterofermentative growth of the plant isolate Lactobacillus florum 2F. J Appl Microbiol 120:13361345. https://doi.org/10.1111/jam.13108 